\title{
Representation of Radon shape diffusions via hyperspherical Brownian motion
}

\author{
BY VICTOR M. PANARETOS $\dagger$ \\ Department of Statistics, University of California, Berkeley, U.S.A. \\ e-mail: victorestat. berkeley.edu
}

(Received 14 April 2006; revised 25 October 2007)

\begin{abstract}
A framework is introduced for the study of general Radon shape diffusions, that is, shape diffusions induced by projections of randomly rotating shapes. This is done via a convenient representation of unoriented Radon shape diffusions in (unoriented) D.G. Kendall shape space $\widetilde{\Sigma}_{n}^{k}$ through a Brownian motion on the hypersphere. This representation leads to a coordinate system for the generalized version of Radon diffusions since it is shown that shape can be essentially identified with unoriented shape in the projected case. A bijective correspondence between Brownian motion on real projective space and Radon shape diffusions is established. Furthermore, equations are derived for the general (unoriented) Radon diffusion of shape-and-size, and stationary measures are discussed.
\end{abstract}

Dedicated to the memory of John Trevor Lewis (1932-2004)

1. Introduction: shape theory and the diffusion of Radon shape

Although everyone seems to have an intuitive grasp of what shape is, shape is a vague concept (using Tukey's terminology, e.g. [14]), in the sense that its meaning can be made precise in many ways. The statistical theory of shape introduced by D.G. Kendall (Kendall et al. [7]; Dryden and Mardia [4]; Small [20]; Stoyan et al. [21]) is a mathematical approach that makes the meaning of shape precise and enables the statistical study of shape in data. Informally, the shape of a data set is the set of those characteristics of the data that are invariant under rotations, translations and scaling. More concretely, consider a set of not totally coincident labelled points $\left\{\mathbf{x}_{m}\right\}_{m=1}^{k}$ in Euclidean space $\mathbb{R}^{n}$, centred to have centroid zero and scaled to have total size unity:

$$
\sum_{m=1}^{k} \mathbf{x}_{m}=\mathbf{0} \text { and }\left\{\sum_{m=1}^{k}\left\|\mathbf{x}_{m}\right\|^{2}\right\}^{\frac{1}{2}}=1 .
$$

Then, the shape of the ensemble $\left\{\mathbf{x}_{m}\right\}_{m=1}^{k}$ is its orbit under the group action of $\mathrm{SO}(n)$ acting from the left (the left orbits of $\mathrm{O}(n)$ are the so-called unoriented shape or reflection shape or modified shape). Under this definition, the shapes of such ensembles can be thought of

† Now at: Institute of Mathematics, Ecole Polytechnique Fédérale de Lausanne, Switzerland, e-mail: victor.panaretos@epfl.ch 
as points on a manifold, so as to allow the definition of statistical procedures that assess, compare and estimate shape characteristics. The geometry of shape spaces $\Sigma_{n}^{k}$ of $k$ labelled points in $n$ dimensions varies for different values of $k$ and $n$ and can be rather intricate (Kendall et al. [7], Le and Kendall [12], Carne [1], Le [11]).

The D.G. Kendall school of shape is thought to originate from a consideration put forward by D.G. Kendall [6]: if $k$ labelled points perform independent Brownian motions in $\mathbb{R}^{n}$, what is the induced process their shape performs? It was shown by D.G. Kendall that for planar ensembles the resulting process is a time-changed Brownian motion in shape space (indicating how shape spaces are connected to the Brownian motion of landmarks; see also W.S. Kendall [10]).

A different type of diffusion of shape within the D.G. Kendall framework was introduced by the author [16], motivated from problems in structural biology (see Section 2.2). Consider, in particular, an arrangement of labelled points on the plane, and assume that these are being rotated randomly, as a Brownian motion on $\mathrm{SO}(2)$ acts on each of them from the left (that is, as it acts on each column vector). The shape of the resulting planar diffusion remains invariant in time, but the same is not true for the shape of its projection onto a fixed line. The resulting shape diffusion was termed a Radon diffusion of shape, drawing an analogy to the Radon transform.

It was shown in [16] that when the columns of $V$ are not collinear, the shape of the projection evolves as a diffusion whose support is a great circle of the unit hypersphere. In addition, it was seen that the stationary distribution of the shape diffusion with respect to Lebesgue measure on the particular great circle was connected to the central angular Gaussian class (see Watson [23]) characterized by a matrix parameter that is in bijective correspondence with the unoriented shape of $V$. This result can be viewed as a stochastic analogue of the deterministic case of Radon transforms, within the framework of shape theory: knowledge of the distribution of the shape of projections over random angles determines the shape of the generating ensemble (so that a shape is connected with a shape).

This paper introduces a framework for the study of Radon diffusions in general shape space $\Sigma_{n}^{k}$. In particular, we observe that the distinction between proper shape and unoriented shape is artificial in the case of projections, and employ an inner product coordinate system. It is seen that this coordinate system is quite convenient and can be made to depend bijectively on a Brownian motion on a hypersphere of appropriate dimension. Intuitively, this reduces to the fact that by rotational invariance, it makes no difference if we rotate an ensemble and project it onto a fixed plane or keep the ensemble at a fixed place and project it onto randomly moving planes.

Before introducing the general case of Radon shape diffusions, we recall the definition of inner product coordinates (see also W.S. Kendall [9]). The inner product coordinates for the unoriented shape of a centred ensemble $\left\{\mathbf{x}_{m}\right\}_{m=1}^{k} \subset \mathbb{R}^{n}$ of unit size are given by the matrix

$$
\mathcal{S}:=\left(\begin{array}{cccc}
\left\|\mathbf{x}_{1}\right\|^{2} & \left\langle\mathbf{x}_{1}, \mathbf{x}_{2}\right\rangle & \ldots & \left\langle\mathbf{x}_{1}, \mathbf{x}_{k}\right\rangle \\
\left\langle\mathbf{x}_{2}, \mathbf{x}_{1}\right\rangle & \left\|\mathbf{x}_{2}\right\|^{2} & \ldots & \vdots \\
\vdots & \vdots & \ddots & \left\langle\mathbf{x}_{k-1}, \mathbf{x}_{k}\right\rangle \\
\left\langle\mathbf{x}_{k}, \mathbf{x}_{1}\right\rangle & \ldots & \left\langle\mathbf{x}_{k}, \mathbf{x}_{k-1}\right\rangle & \left\|\mathbf{x}_{k}\right\|^{2}
\end{array}\right)
$$

whose $i j$ th element is the inner product $\left\langle\mathbf{x}_{i}, \mathbf{x}_{j}\right\rangle$. Thus, $\mathcal{S}$ provides all the norms of the vectors defining the ensemble, along with their pairwise angles, encoding their unoriented shape 
(since the size is assumed to be unity, and the centroid is assumed to be zero). Notice the straightforward but important fact that these coordinates are invariant under imbedding. By this it is meant, that if $\mathbb{R}^{n}$ is imbedded as any $n$-dimensional hyperplane into $\mathbb{R}^{N}, N>n$, then the inner product coordinates will be valid as a parametrization of the unoriented shape of $\left\{\mathbf{x}_{m}\right\}_{m=1}^{k}$ as an element of $\Sigma_{n}^{k}$. Furthermore, the inner product coordinates will parameterize the proper shape (i.e. not unoriented) of $\left\{\mathbf{x}_{m}\right\}_{m=1}^{k}$ as an element of $\Sigma_{N}^{k}$, since any reflection of points living in a subspace of dimension $n<N$ can be carried out by a rotation in $\mathrm{SO}(N)$.

The paper is organized as follows. Section 2 introduces notation and the general framework. Section 3 provides a setup for Radon shape diffusions in arbitrary dimensions. This is done in two ways, the first using a Brownian motion in the rotation group $\mathrm{SO}(n)$ and the second using a Brownian motion on the unit hypersphere $\mathbb{S}^{n-1}$. It is shown that although the two approaches are equivalent, the second approach has important advantages. In Section 4, the representation through a hyperspherical Brownian motion is employed to derive certain basic results on general unoriented Radon shape diffusions. It is demonstrated that Radon shape processes are diffusions in the general case, and that they are in bijective correspondence with Brownian motion on real projective space (of appropriate dimension). Equations are obtained for the shape-and-size process, and aspects of the stationary distributions are explored. The paper closes with a brief discussion in Section 5.

\section{The shape of projections}

\subsection{Assumptions and Notation}

Consider a labelled ensemble formed by $k$ labelled vertices $\left\{\mathbf{x}_{i}, 1 \leqslant i \leqslant k\right\}$ in $n$ dimensional Euclidean space $\mathbb{R}^{n}$ (with $n<k$ ). We call this vertex ensemble the generating ensemble, and take the ordering of the vertices to correspond to their labels. We may arrange the vertex set of this generating ensemble as the columns of an $n \times k$ matrix $V$ :

$$
V:=\left(\begin{array}{llll}
\mathbf{x}_{1} & \mathbf{x}_{2} & \ldots & \mathbf{x}_{k}
\end{array}\right) .
$$

Thus, the column arrangement provides the labels corresponding to each point.

Notice that we have assumed that the number of elements is $k>n$ for dimension $n$. In addition, we shall assume that the vertex set $V$ is not of an effectively lesser dimension (and call it proper), in the sense that there is no $(n-1)$-dimensional hyperplane containing all of the $k$ vertices. These assumptions aim at limiting us to situations where the shape characteristics of $V$ are genuinely $n$-dimensional. Since the location of $V$ is not of interest, we may further assume that the centroid of the generating ensemble is zero, i.e. the row sums of $V$ are zero.

When we rotate the ensemble according to a rotation matrix $\Phi \in \mathrm{SO}(n)$, we obtain the ensemble $\Phi V$. The projection of the rotated ensemble onto the hyperplane $\mathcal{H}:=$ $\left\{\left(x_{1}, \ldots, x_{n}\right) \in \mathbb{R}^{n}: x_{n}=0\right\}$ can then be regarded as an ensemble in $\mathbb{R}^{n-1}$, given by the vertex matrix

$$
p(V, \Phi)=H \Phi V
$$

where $H$ is defined as

$$
\underset{(n-1) \times n}{H}:=\left(I_{n-1} \mathbf{0}_{n-1}\right),
$$

with $I_{n-1}$ denoting the $(n-1)$-dimensional identity matrix and $\mathbf{0}_{n-1}$ denoting an $(n-1)$ vector of zeroes. 
The pre-shape of $p(V, \Phi)$ is its orbit under the scaling group and its representation can be obtained as

$$
s_{t}=\frac{H \Phi V}{\sqrt{\operatorname{trace}\left(V^{\top} \Phi^{\top} H^{\top} H \Phi V\right)}} .
$$

We quotient out from the left by the group $\mathrm{O}(n-1)$ in order to obtain the unoriented shape of the projected vertex ensemble. The resulting equivalence classes are naturally parameterized through the inner product coordinates

$$
\widetilde{\sigma}_{t}=s_{t}^{\top} s_{t}=\frac{V^{\top} \Phi^{\top} H^{\top} H \Phi V}{\operatorname{trace}\left(V^{\top} \Phi^{\top} H^{\top} H \Phi V\right)} .
$$

The unoriented shape-and-size is parameterized by

$$
\widetilde{S}_{t}=V^{\top} \Phi^{\top} H^{\top} H \Phi V .
$$

In order to obtain the proper shape $\sigma$ (and shape-and-size $S$ ), one has to couple $\widetilde{\sigma}$ (and $\widetilde{S}$, respectively) with an indicator of the orientation of the column-space of $p(V, \Phi)$, so as to discriminate between rotoinversions.

\subsection{A biophysical motivation}

Consider a biological particle moving freely in an aqueous environment. A simple model for this movement is a Brownian motion in $\mathbb{R}^{3}$. Suppose though, that we do not want to think of the particle as a single point in space, but want to model the movement of the actual particle, which is an ensemble of different sub-parts (molecular assemblies). Specifically, we choose $k$ physically significant labelled loci on the particle (these could be molecular assemblies, molecules, or even atoms) and suppose that the particle is characterized by the shape of these loci. A simple model for the movement of the particle in its aqueous environment would be

$$
V_{t}=\Phi_{t} V_{0}+B_{t} \mathbf{1}_{k}^{\top}
$$

where $V_{0}$ is a $3 \times k$ matrix whose columns give the initial position of the characterising loci, $\left\{\Phi_{t}\right\}$ is a rotational Brownian motion (see Section 3 ), $\left\{B_{t}\right\}$ is a Brownian motion in $\mathbb{R}^{3}$, independent of $\left\{\Phi_{t}\right\}$, and $\mathbf{1}_{k}$ is a $k$-vector with all of its entries equal to unity. Naturally, this sort of movement leaves the shape-and-size of the particle unchanged. However, if we were able to observe the movement of this particle through a microscope, we would be observing its projection on the observation plane,

$$
H V_{t}=H \Phi_{t} V_{0}+H B_{t} \mathbf{1}_{k}^{\top},
$$

whose shape-and-size would constantly be changing in time. We would then be interested in inferring the structural properties of $V_{0}$ from the observation of $H V_{t}$. Of course, neither the location of the projected particle, nor its orientation provide information on the original particle $V$. However, the shape characteristics should be providing information, and it would be of interest to study their evolution.

A real analogue of this idealised problem arises in single particle biophysics, and, in particular, in the structural biology of macromolecular assemblies. There are various ways to image a biological particle, and one such way is through cryo-electron microscopy (see Glaeser [2]; Glaeser et al. [3]). This method provides images of the particles under observation that are projections (in the sense of line integrals) of the actual particles. If one is not imaging a crystalline structure, then it is impossible to rotate the particles at will so as to 
directly employ Radon-transform techniques. In particular, the particles (which are assumed to be identical) are allowed to move freely in an aqueous environment and are frozen at some random positions and orientations, so that the data are projections of (many) particles at random positions and under varying random orientations. The data must then be used to extract structural information on the original particle.

\subsection{The (un)importance of the orientation of projections}

The shape of a Radon diffusion has a special nature because it always makes reference to a generating ensemble $V$. Although the strict definition of shape requires that we distinguish between rotoinversions, such a distinction is artificial in the case of Radon diffusions. This is because rotoinversions of projections always occur by rotations of $V$ at "opposite" angles:

LEMMA 1. Let $\sigma_{1}, \sigma_{2} \in \Sigma_{n-1}^{k}$ be two shapes corresponding to the projections $p\left(V, \Phi_{1}\right)$ and $p\left(V, \Phi_{2}\right)$, for some proper generating ensemble $V$ and $\Phi_{1}, \Phi_{2} \in \operatorname{SO}(n)$. Then $\sigma_{1}$ is a reflection of $\sigma_{2}$ if and only if

$$
\Phi_{1}=\left(\begin{array}{cc}
R & \mathbf{0}_{n-1} \\
\mathbf{0}_{n-1}^{\top} & -1
\end{array}\right) \Phi_{2}
$$

for some $R \in \mathrm{O}(n-1) \backslash \mathrm{SO}(n-1)$, and $\mathbf{0}_{n-1}$ as before.

Proof. Assume that $\sigma_{1}$ is a reflection of $\sigma_{2}$. This implies that $p\left(V, \Phi_{1}\right)=c B p\left(V, \Phi_{2}\right)$ for some $B \in \mathrm{O}(n-1) \backslash \mathrm{SO}(n-1)$ and some scalar $c>0$, so that the corresponding unoriented shapes, $\widetilde{\sigma}_{1}$ and $\widetilde{\sigma}_{2}$, are equal. Hence, the corresponding parametrizations must be equal:

$\tilde{\sigma}_{1}=\widetilde{\sigma}_{2} \Longrightarrow \frac{V^{\top} \Phi_{1}^{\top} H^{\top} H \Phi_{1} V}{\operatorname{tr}\left(V^{\top} \Phi_{1}^{\top} H^{\top} H \Phi_{1} V\right)}=\frac{V^{\top} \Phi_{2}^{\top} H^{\top} H \Phi_{2} V}{\operatorname{tr}\left(V^{\top} \Phi_{2}^{\top} H^{\top} H \Phi_{2} V\right)} \Longrightarrow \Phi_{1}^{\top} H^{\top} H \Phi_{1}=\frac{\Phi_{2}^{\top} H^{\top} H \Phi_{2}}{c^{2}}$,

where $c^{2}$ is the ratio of the two sizes (the final implication follows from the assumption that $V$ is a proper generating ensemble). Since the left-hand side in the final equality is an idempotent matrix, it follows that $c=1$ (i.e. the sizes are the same).

Let $\mathcal{M}\left(\Phi_{1}^{\top} H^{\top}\right)$ denote the column space of $\Phi_{1}^{\top} H^{\top}$. Then the projection onto $\mathcal{M}\left(\Phi_{1}^{\top} H^{\top}\right)$ is given by

$$
\Phi_{1}^{\top} H^{\top}\left(H \Phi_{1} \Phi_{1}^{\top} H^{\top}\right)^{-1} H \Phi_{1}=\Phi_{1}^{\top} H^{\top} H \Phi_{1}
$$

Similarly, the projection matrix onto $\mathcal{M}\left(\Phi_{2}^{\top} H^{\top}\right)$ is given by $\Phi_{2}^{\top} H^{\top} H \Phi_{2}$. It follows from relation $(2 \cdot 10)$ that

$$
\operatorname{span}\left(\phi_{1}^{(1)}, \ldots, \phi_{n-1}^{(1)}\right)=\mathcal{M}\left(\Phi_{1}^{\top} H^{\top}\right)=\mathcal{M}\left(\Phi_{2}^{\top} H^{\top}\right)=\operatorname{span}\left(\phi_{1}^{(2)}, \ldots, \phi_{n-1}^{(2)}\right),
$$

where $\phi_{j}^{(i)}$ is the $j$ th row $(1 \leqslant j \leqslant n)$ of $\Phi_{i}, i=1,2$.

But $\Phi_{1}$ and $\Phi_{2}$ are special orthogonal matrices, hence they both have determinant 1 . This means that there is a dichotomy between the following two cases:

(i) we have $\phi_{n}^{(1)}=\phi_{n}^{(2)}$ and $\left(\phi_{1}^{(1)}, \ldots, \phi_{n-1}^{(1)}\right)$ is transformed into $\left(\phi_{1}^{(2)}, \ldots, \phi_{n-1}^{(2)}\right)$ by a rotation around the axis defined by $\phi_{n}^{(1)}$,

(ii) we have $\phi_{n}^{(1)}=-\phi_{n}^{(2)}$ and $\left(\phi_{1}^{(1)}, \ldots, \phi_{n-1}^{(1)}\right)$ is transformed into $\left(\phi_{1}^{(2)}, \ldots, \phi_{n-1}^{(2)}\right)$ by a rotoinversion around the axis defined by $\phi_{n}^{(1)}$.

Assume the first case is true. Then, we may write

$$
H \Phi_{1} V=H\left(\begin{array}{cc}
K & \mathbf{0}_{n-1} \\
\mathbf{0}_{n-1}^{\top} & 1
\end{array}\right) \Phi_{2} V=K H \Phi_{2} V
$$




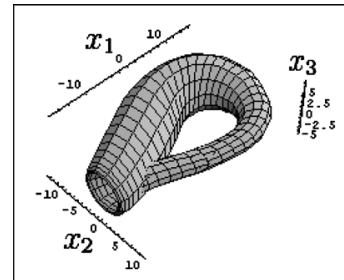

(1)

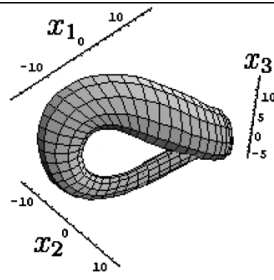

(2)

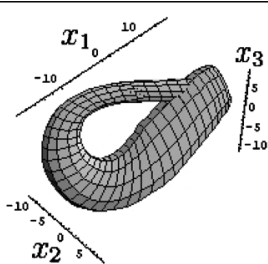

(3)

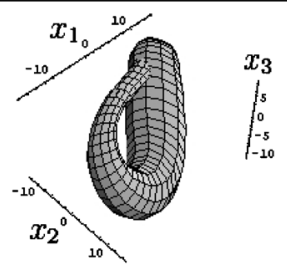

(4)

Fig. 1. Schematic representation of the rotation of a Klein bottle (object (1)) according to $\Phi_{2}$ and $\Phi_{1}$, when these are related according to equation (2.9). Bottle (2) is the result of the action of $\Phi_{2}$ on bottle (1). Bottle (3) is the result of the action of $\Phi_{1}$, for for $R=\operatorname{diag}\{1,-1\}$, on bottle (1). Bottle (4) in the result of the action of $\Phi_{1}$ on bottle (1), for a different choice of $R$, namely being the composition of $\operatorname{diag}\{1,-1\}$ with a clockwise rotation by $\pi / 3$ radians.

for some $K \in \mathrm{SO}(n-1)$, which contradicts our assumption that $\sigma_{1} \neq \sigma_{2}$. Therefore, it must be that the second case holds, which is equivalent to equation (2.9).

To establish the converse, assume that (2.9) holds. Then it suffices to observe that,

$$
H \Phi_{1} V=H\left(\begin{array}{cc}
R & \mathbf{0}_{n-1} \\
\mathbf{0}_{n-1}^{\top} & -1
\end{array}\right) \Phi_{2} V=R H \Phi_{2} V,
$$

so that $p\left(V, \Phi_{1}\right)=R p\left(V, \Phi_{2}\right)$ for some $R \in \mathrm{O}(n-1) \backslash \mathrm{SO}(n-1)$, and the proof is complete.

This shows that reflected Radon shapes can only occur as projections of the generating ensemble at 'opposite' rotations. In particular, for two dimensional ensembles, this means that $\sigma_{1}$ is a reflection of $\sigma_{2}$ if and only if $\Phi_{1}=e^{i \theta}$ and $\Phi_{2}=e^{-i \theta}$. For three dimensional ensembles, $\sigma_{1}$ is a reflection of $\sigma_{2}$ if and only if the $\Phi_{1}$ rotation works as follows: first it rotates the space according to $\Phi_{2}$. Then it reflects the space with respect to the plane $x_{3}=0$. Finally, it performs a rotoinversion (improper rotation) with respect to the the $x_{3}$-axis. The result of the action of $\Phi_{1}$ is an 'upside-down' version of the result of the action of $\Phi_{2}$, possibly with an additional (proper) rotation around the $x_{3}$-axis (see Figure 1).

As such, the distinction between rotoinversions does not yield any additional information about the structure of the generating ensemble. Distinguishing between unoriented shape and proper shape introduces a redundancy, that only makes the geometry of the problem more complicated. We shall therefore only consider unoriented shape (i.e. we will quotient out by $\mathrm{O}(n-1)$ rather than by $\mathrm{SO}(n-1))$. In some sense, when dealing with projected ensembles, shape coincides with unoriented shape This is in analogy with the fact that, since any reflection in $\mathbb{R}^{n-1}$ can be realized as a rotation in $\mathbb{R}^{n}$, the unoriented shape of a projection coincides with its shape when imbedded in $\mathbb{R}^{n}$. Consequently, we shall restrict our attention to the study of unoriented shape-and-size and unoriented shape.

\section{Representations for general Radon diffusions}

To generalize the planar case, we want to consider the evolution of the unoriented shape of the projection of $V$ onto $\mathcal{H}$ as the former is being diffusively rotated. The approach is to let a Brownian motion in $\mathrm{SO}(n)$ act on $V$ from the left, and observe the unoriented shape characteristics of the projections. There are various approaches to defining Brownian motion on a manifold (e.g. W.S. Kendall [8]). Here, we define $\mathrm{BM}(\mathrm{SO}(n))$ through the so-called product-integral injection. Let $\mathcal{A}(G)$ be the Lie algebra generated by a multiplicative group 
$G$ of $n \times n$ matrices, i.e.

$$
\mathcal{A}(G)=\left\{A \in \mathrm{GL}(n): e^{t A} \in G \forall t \in \mathbb{R}\right\} .
$$

It is possible to define Brownian motion on any such group $G$ by defining Brownian motion on $\mathcal{A}(G)$ through the Stratonovich SDE (Rogers and Williams [19]):

$$
\partial G=G \partial A
$$

where $\{A(t)\}$ is $\operatorname{BM}(\mathcal{A}(G))$.

In the case of the special orthogonal group $\mathrm{SO}(n)$, the Lie algebra $\mathcal{A}(\mathrm{SO}(n))$ is the algebra SK $(n)=\left\{F \in \operatorname{GL}(n): F^{\top}=-F\right\}$ of skew symmetric $n \times n$ matrices. We first define Brownian motion on $\operatorname{SK}(n)$. To do this, let $F(m, k)=\left\{f_{i j}(m, k)\right\} \in \operatorname{SK}(n)$ be defined as

$$
f_{i j}(m, k)= \begin{cases}1, & \text { if } \quad(i, j)=(m, k) \\ -1, & \text { if } \quad(i, j)=(k, m) \\ 0, & \text { otherwise. }\end{cases}
$$

Then, the collection $\{F(m, k)\}_{m<k}$ constitutes a basis for $\operatorname{SK}(n)$, which allows us to define $\operatorname{BM}(\operatorname{SK}(n))$ as

$$
A_{t}:=\sum_{1 \leqslant m<k \leqslant n} F(m, k) B_{t}(m, k),
$$

where $\left\{B_{t}(m, k)\right\}_{m<k}$ is a collection of independent standard Brownian motions on $\mathbb{R}$.

A (bi-invariant) Brownian motion $\Phi_{t}$ on $\mathrm{SO}(n)$ will thus be the solution to the Stratonovich equation

$$
\partial \Phi_{t}=\Phi_{t} \partial A_{t} .
$$

We are now in a position to define the process of the diffusively rotated and projected vertex ensemble,

$$
p\left(V, \Phi_{t}\right)=H \Phi_{t} V,
$$

where $H$ is as before. The unoriented shape and unoriented shape-and-size processes will thus be

$$
\widetilde{\sigma}_{t}=\frac{V^{\top} \Phi_{t}^{\top} H^{\top} H \Phi_{t} V}{\operatorname{trace}\left(V^{\top} \Phi_{t}^{\top} H^{\top} H \Phi_{t} V\right)}
$$

and

$$
\widetilde{S}_{t}=V^{\top} \Phi_{t}^{\top} H^{\top} H \Phi_{t} V
$$

respectively. Thus, it may be suggested that the unoriented Radon shape diffusion is apparently not very usefully represented through a Brownian motion in the rotation group $\mathrm{SO}(n)$. One can argue that the approach is rather abstract (involving a Brownian motion on a matrix group), and that it does not provide a good means for simulation. It would seem desirable that we have an alternative representation of the unoriented Radon shape diffusion. One that would be equivalent but more straightforward. Already, the form $\Phi_{t}^{\top} H^{\top} H \Phi_{t}$ can be recognized as a spectral decomposition of some projection matrix of rank $n-1$, providing an indication that this should be possible.

So, let us adopt a slightly different viewpoint. Rather than study the evolution of the projections of $V$ onto a fixed hyperplane of dimension $n-1$, as $\mathrm{BM}(\mathrm{SO}(n))$ acts on $V$, we consider the process that results from projecting $V$ onto a randomly moving hyperplane. The motion of the hyperplane will be such that it is normal to a Brownian motion on the 
unit hypersphere $\mathbb{S}^{n-1}$. To be more precise, if $\left\{u_{t}\right\}_{t \geqslant 0}$ is a Brownian motion on $\mathbb{S}^{n-1}$, the hyperplane normal to $u_{t}$ at time $t$ is

$$
P_{t}:=\left\{\mathbf{x} \in \mathbb{R}^{n}: \mathbf{x}^{\top} u_{t}=0\right\}
$$

and it follows that the projection operator onto the plane $P_{t}$ is given by the $n \times n$ matrix

$$
\Pi_{t}:=I-u_{t} u_{t}^{\top} .
$$

The projection of the ensemble $V$ is a $k$-ad of vectors contained in the hyperplane $P_{t}$, given by the columns of the $n \times k$ matrix $\Pi_{t} V$. The centroid of these vectors is zero by assumption, so that the inner product coordinates for their unoriented shape-and-size are given by

$$
\widetilde{S}_{t} \equiv \widetilde{S}\left(\Pi_{t} V\right)=V^{\top} \Pi_{t} V,
$$

upon recalling that the projection $\Pi_{t}$ is idempotent. Accordingly, the unoriented shape is parameterized by its inner product coordinates,

$$
\widetilde{\sigma}_{t} \equiv \widetilde{\sigma}\left(\Pi_{t} V\right)=\frac{V^{\top} \Pi_{t} V}{\operatorname{tr}\left(V^{\top} \Pi_{t} V\right)} .
$$

This representation is precisely equivalent to the original formulation, but simplifies things by taking advantage of the special properties of shape:

PROPOSITION 1. The unoriented Radon shape diffusions resulting from rotational Brownian motion are equivalent to those resulting from hyperspherical Brownian motion.

Proof. Consider the action of $\left\{\Phi_{t}^{\top}\right\}$ on the north pole $\rho:=(0,0, \ldots, 1)^{\top}$, say $Y_{t}=\Phi_{t}^{\top} \rho$. By equation $(3 \cdot 5)$,

$$
\partial Y_{t}=\partial\left(\Phi_{t}^{\top} \rho\right)=\left(\partial \Phi_{t}\right)^{\top} \rho=\left(\partial A_{t}\right)^{\top} \Phi_{t}^{\top} \rho=\left(\partial A_{t}^{\top}\right) Y_{t},
$$

where $\left\{A_{t}^{\top}\right\}$ is again a Brownian motion on the skew-symmetric group. The equation $\partial Y_{t}=$ $\left(\partial A_{t}^{\top}\right) Y_{t}$ gives a Brownian motion on the hypersphere (Van Den Berg and Lewis [22]). Moreover, consider the projection onto the plane normal to $Y_{t}$. This will be the projection $\mathcal{P}$ onto the span of the first $n-1$ columns of $\Phi_{t}^{\top}$, that is, the projection onto the columnspace of $\Phi_{t}^{\top} H^{\top}$

$$
\mathcal{P}=\Phi_{t}^{\top} H^{\top}\left(H \Phi_{t} \Phi_{t}^{\top} H^{\top}\right)^{-1} H \Phi_{t}=\Phi_{t}^{\top} H^{\top} H \Phi_{t} .
$$

But $\mathcal{P}=I-Y_{t} Y_{t}^{\top} \stackrel{d}{=} I-\beta_{t} \beta_{t}^{\top}$, for $\left\{\beta_{t}\right\}$ a hyperspherical Brownian motion started at $\beta_{0}=\rho$, and so,

$$
V^{\top} \Phi_{t}^{\top} H^{\top} H \Phi_{t} V \stackrel{d}{=} V^{\top} \Pi_{t} V, \quad \forall t \geqslant 0 .
$$

This completes the proof.

Of course, the two approaches are far from being equivalent as far as the actual projections are concerned. For one, the projections in the first case $(\operatorname{BM}(\operatorname{SO}(n)))$ always live in the same subspace, namely $\mathcal{H}$, whereas in the second case $\left(\mathrm{BM}\left(\mathbb{S}^{n-1}\right)\right)$, the projections live in constantly changing hyperplanes. Furthermore, the $\mathrm{BM}(\mathrm{SO}(n))$ approach allows for the possibility of rotations within the projection hyperplane, while for the $\operatorname{BM}\left(\mathbb{S}^{n-1}\right)$, once a projection hyperplane has been fixed, there can only be one projection within it. Naturally, once we talk about shape, these distinctions make no difference. The first approach is the exact translation of the physical situation, where the observation plane is fixed and the particle 
is being randomly rotated. However, once we talk about shape, the precise description that requires a fixed plane and the possibility of within-plane rotations is unnecessary, and, in fact, only complicates things.

\section{Stochastic differential equations and stationary distributions}

When the original ensemble is planar, its projections are one dimensional and one is able to take advantage of the metric equivalence of the shape space $\Sigma_{1}^{k}$ to a sphere of appropriate dimension. In the case of general shape spaces $\Sigma_{n}^{k}$, it seems not possible to find similarly simple representations. In fact, the differential geometry of these spaces had not appeared in earlier contexts and, as noted in the introduction, can be rather involved. However, some questions can be answered within the context provided by the inner product representations of the unoriented shape and unoriented shape-and-size (equations (3.12) and (3.11), respectively). For example, suppose we are able to observe $\left\{\widetilde{\sigma}_{t}\right\}_{t=t_{0}}^{t_{1}}$ and have knowledge of $V$. Is it possible to recover the process of projection hyperplanes? If yes, is this true for all possible $V$ ? The answer is given in the following proposition.

PROPOSITION 2. Assume that $V$ is known and that a sample path of $\left\{\widetilde{\sigma}_{t}\right\}$ is available. Then, it is possible to recover $\left\{u_{t}\right\}$ up to a sign change if and only if $V$ is of rank $n$ (i.e. if and only if $V$ is proper).

Proof. Fix $t \in\left(t_{0}, t_{1}\right)$. Since $t$ is fixed, we suppress the time index. For tidiness, define $\xi:=\operatorname{tr}\left(V^{\top} \Pi V\right)>0$ and $\Pi^{\diamond}:=\Pi / \xi$. Then, we observe

$$
\tilde{\sigma}=V^{\top} \Pi^{\diamond} V .
$$

Suppose we are able to recover $\Pi^{\diamond}$. Since $\Pi$ is idempotent, we have that

$$
\left(\Pi^{\diamond}\right)^{r}=\frac{1}{\xi^{r}} \Pi, \quad r=1,2, \ldots
$$

Now $\xi>0$, so the $i$ th element of $\left(\Pi^{\diamond}\right)^{r}$ is non-zero if and only if the respective element of $\Pi^{\diamond}$ is non zero. Let $i, j$ be such that $\left(\Pi^{\diamond}\right)_{i j} \neq 0$ (there necessarily exists such an element by $\Pi$ being a projection onto a subspace of dimension $n-1)$. Then,

$$
\frac{\left(\Pi^{\diamond}\right)_{i j}}{\left(\Pi^{\diamond 2}\right)_{i j}}=\frac{(\Pi)_{i j} \xi^{2}}{(\Pi)_{i j} \xi}=\xi
$$

Knowledge of $\Pi^{\diamond}$ enables us to determine $\Pi$, and hence, $u u^{\top}$. Therefore, we know that $u$ lies on a specific straight line through the origin, and by $\|u\|=1$ we can determine $u$ as one of two antipodal points on $\mathbb{S}^{n-1}$.

It remains to determine necessary and sufficient conditions for the unique recovery of $\Pi^{\diamond}$. The determination of $\Pi^{\diamond}$ requires the solution of

$$
\tilde{\sigma}=V^{\top} \Pi^{\diamond} V .
$$

This is a matrix equation for the unknown matrix $\Pi^{\diamond}$. Such an equation may be transformed into a vector equation for the elements of the vectorised version of $\Pi^{\diamond}$,

$$
\left(V^{\top} \otimes V^{\top}\right) \operatorname{vec}\left(\Pi^{\diamond}\right)=\operatorname{vec}(\tilde{\sigma}),
$$

where "vec" is the vectorising operator (by column) and " $\otimes$ " is the Kronecker product. The system of equations (4.5) contains $k^{2}$ equations for $n^{2}$ unknowns, and in general is 
overdetermined, since $k>n$. However, we know that it has a solution. Hence, the system has $n^{2}$ consistent equations and a there is a dichotomy between the existence of a unique solution (if the rank of the system is $n^{2}$ ), or of infinitely many solutions (if the rank is reduced). Of course, the rank of the system is equal to the rank of $V^{\top} \otimes V^{\top}$, with

$$
\operatorname{rank}\left(V^{\top} \otimes V^{\top}\right)=\operatorname{rank}\left(V^{\top}\right)^{2} .
$$

Summarising, if $\operatorname{rank}(V)=n$, the system has a unique solution. If $V$ is of reduced row rank, the system has infinitely many solutions. In particular, the solution in the former case will be

$$
\Pi^{\diamond}=\left(V V^{\top}\right)^{-1} V \tilde{\sigma} V^{\top}\left(V V^{\top}\right)^{-1}
$$

This completes the proof.

The possibility of such a recovery enables us to proceed with the questions of whether $\left\{\widetilde{S}_{t}\right\}$ and $\left\{\widetilde{\sigma}_{t}\right\}$ are indeed diffusions, and of what can be inferred on the shape of $V$ from observation of a sample path of any of these two.

THEOREM 1. Let $V$ be a proper labelled ensemble consisting of $k$ points in $\mathbb{R}^{n}, k>n$. Let $\Pi_{t}=I-u_{t} u_{t}^{\top}$, where $\left\{u_{t}\right\}$ is Brownian motion on the unit hypersphere $\mathbb{S}^{n-1}$, and $\left\{B_{t}\right\}$ be standard Brownian motion in $\mathbb{R}^{n}$. Then, the unoriented shape-and-size $\widetilde{S}_{t} \equiv \widetilde{S}\left(\Pi_{t} V\right)$ of the Radon process $\left\{\Pi_{t} V\right\}$ is a diffusion solving the Itô stochastic differential equation

$$
\begin{gathered}
d{\widetilde{S_{t}}}=-V^{\top}\left\{\mathcal{E}\left(\widetilde{S}_{t}\right) d B_{t} \chi\left[\mathcal{E}\left(\widetilde{S}_{t}\right)\right]^{\top}+\chi\left[\mathcal{E}\left(\widetilde{S}_{t}\right)\right] d B_{t}^{\top} \mathcal{E}\left(\widetilde{S}_{t}\right)^{\top}\right\} V \\
+V^{\top}\left\{(n-1)\left(I-\mathcal{E}\left(\widetilde{S}_{t}\right)\right)-\mathcal{E}\left(\widetilde{S}_{t}\right)\right\} V d t,
\end{gathered}
$$

where $\mathcal{E}\left(\widetilde{S}_{t}\right)=\left(V V^{\top}\right)^{-1} V \widetilde{S}_{t} V^{\top}\left(V V^{\top}\right)^{-1}$, and $\chi[\Pi]$ signifies the unit vector of positive orientation defined by the line on which $I-\Pi$ projects.

Proof. Since $\widetilde{S}_{t}=V^{\top} \Pi_{t} V$, we first concentrate on $\left\{\Pi_{t}\right\}$. Recall that

$$
\Pi_{t}:=I-u_{t} u_{t}^{\top} .
$$

The process $\left\{u_{t}\right\}$ is a Brownian motion on the unit hypersphere $\mathbb{S}^{n-1}$, solving the Itô stochastic differential equation (e.g. Øksendal [15])

$$
d u_{t}=-\left(\frac{n-1}{2}\right) u_{t} d t+\left(I-u_{t} u_{t}^{\top}\right) d \breve{B}_{t},
$$

where $\left\{\breve{B}_{t}\right\}$ is standard Brownian motion in $\mathbb{R}^{n}$.

Let $g(x)=I-x x^{\top}$ for $x \in \mathbb{R}^{n}$. The mapping $g: \mathbb{R}^{n} \rightarrow \mathbb{R}^{n^{2}}$ is twice continuously differentiable everywhere on $\mathbb{R}^{n}$. Since $\Pi_{t}=g\left(u_{t}\right)$ we may apply the multidimensional Itô lemma to obtain the stochastic differential equation satisfied by the $m k$ th element of $\Pi_{t}$ :

$$
d \Pi_{m k}=\sum_{i=1}^{n} \frac{\partial g_{m k}}{\partial u_{i}} d u_{i}+\frac{1}{2} \sum_{i=1}^{n} \sum_{j=1}^{n} \frac{\partial^{2} g_{m k}}{\partial u_{i} \partial u_{j}} d u_{i} d u_{j}
$$

where $g(u)=\left\{g_{m k}(u)\right\}_{m, k=1}^{n}$, and the time index has been suppressed for tidiness.

One can see that the first term on the right-hand side of (4.11) may be obtained as the $\{n(k-1)+m\}$-element of,

$$
\left\{\frac{d}{d u} \operatorname{vec}\left(I-u u^{\top}\right)\right\} d u=-(u \otimes I+I \otimes u) d u,
$$


which reduces to (using equation $4 \cdot 10$ )

$$
-\left(u_{t} \otimes I+I \otimes u_{t}\right) \Pi_{t} d \breve{B}_{t}+\frac{n-1}{2}\left(u_{t} \otimes I+I \otimes u_{t}\right) u_{t} d t .
$$

Shifting attention to the second term on the right-hand side of $(4 \cdot 11)$, we notice that $d u_{i} d u_{j}$ is the $i j$ th element of the matrix $d u d u^{\top}$, which in turn is equal to $\Pi d t$ (by equation (4.10)). Keeping in mind that $\Pi$ is symmetric, we have

$$
\begin{aligned}
\frac{1}{2} \sum_{i=1}^{n} \sum_{j=1}^{n} \frac{\partial^{2} g_{m k}}{\partial u_{i} \partial u_{j}} d u_{i} d u_{j} & =\frac{1}{2} d t \sum_{i=1}^{n} \sum_{j=1}^{n} \frac{\partial^{2} g_{m k}}{\partial u_{i} \partial u_{j}} \Pi_{i j} \\
& =\frac{1}{2} d t \sum_{i=1}^{n} \sum_{j=1}^{n} \frac{\partial^{2}\left(-u_{m} u_{k}\right)}{\partial u_{i} \partial u_{j}} \Pi_{i j} \\
& =-\frac{1}{2} d t \sum_{i=1}^{n} \sum_{j=1}^{n}\left(\delta_{i m} \delta_{j k}+\delta_{i k} \delta_{j m}\right) \Pi_{i j} \\
& =-\frac{1}{2} d t\left(\Pi_{m k}+\Pi_{k m}\right) \\
& =-\Pi_{m k} d t
\end{aligned}
$$

where $\delta_{i j}$ is the Kronecker delta function. Therefore, the equations in (4.11) may be expressed via a single vector equation,

$$
d \operatorname{vec}\left(\Pi_{t}\right)=-\left(u_{t} \otimes I+I \otimes u_{t}\right) \Pi_{t} d \breve{B}_{t}+\frac{n-1}{2}\left(u_{t} \otimes I+I \otimes u_{t}\right) u_{t} d t-\operatorname{vec}\left(\Pi_{t}\right) d t .
$$

But, notice that $\left(u_{t} \otimes I\right) u_{t}=\left(I \otimes u_{t}\right) u_{t}=\operatorname{vec}\left(u_{t} u_{t}^{\top}\right)=\operatorname{vec}\left(I-\Pi_{t}\right)$. Leting $\zeta_{t}:=$ $-\left(u_{t} \otimes I+I \otimes u_{t}\right) \Pi_{t}$, we can see that $\zeta_{t} \zeta_{t}^{\top}$ is invariant under sign changes of $u_{t}$. As a result of the above considerations, if $X_{t}$ solves the Itô equation

$$
d \operatorname{vec}\left(X_{t}\right)=-\left(\chi\left[X_{t}\right] \otimes I+I \otimes \chi\left[X_{t}\right]\right) X_{t} d B_{t}+(n-1) \operatorname{vec}\left(I-X_{t}\right) d t-\operatorname{vec}\left(X_{t}\right) d t
$$

for $\left\{B_{t}\right\}$ a standard Brownian motion in $\mathbb{R}^{n}$, then $X_{t}$ coincides in law with $\Pi_{t}$.

To complete the proof, we observe that $\operatorname{vec}\left(V^{\top} \Pi_{t} V\right)=\left(V^{\top} \otimes V^{\top}\right) \operatorname{vec}\left(\Pi_{t}\right)$. An application of Itô's lemma along with the fact that $\left(\mathbf{A}^{\top} \otimes \mathbf{C}\right) \operatorname{vec}(\mathbf{B})=\operatorname{vec}(\mathbf{A B C})$ yields equation (4.8), and hence shows that $\left\{\widetilde{S}_{t}\right\}$ is a diffusion.

COROLlaRY 1. The unoriented shape $\widetilde{\sigma}_{t} \equiv \widetilde{\sigma}\left(\Pi_{t} V\right)$ of the Radon process $\left\{\Pi_{t} V\right\}$ is a diffusion.

Proof. This follows from a combination of Proposition 2 and Theorem 1. If one is to apply Itô's lemma to the image of the Itô diffusion $\left\{\widetilde{S}_{t}\right\}$ under the twice continuously differentiable mapping $A \mapsto A / \operatorname{tr}(A)$, it can be seen that all the quantities involved in the drift and diffusion coefficient of the resulting stochastic differential equation may be expressed as functions of $\tilde{\sigma}_{t}$.

The Itô equation for $\left\{\widetilde{\sigma}_{t}\right\}$ can also be obtained, but its form is complicated and, as a result, appears rather uninformative.

Obtaining explicit expressions of stationary distributions for the matrices parameterizing the unoriented shape-and-size and unoriented shape of the Radon processes seems a highly non-trivial task. Indeed, a workable form for the stationary distribution of $\Pi_{t}$ is not known. To outline these problems, recall that the stationary distribution of a Brownian motion on 
the hypersphere $\mathbb{S}^{n-1}$ will be the uniform distribution on $\mathbb{S}^{n-1}$. Hence, determination of the stationary distribution of $\left\{\Pi_{t}\right\}$ amounts to determining the distribution of $G=U U^{\top}$, where $U$ is uniform on $\mathbb{S}^{n-1}$. This can be seen to have characteristic function (Mardia and Khatri [13])

$$
\Psi_{G}(Z)=\mathbb{E}\{\exp [i \operatorname{tr}(Z G)]\}={ }_{1} F_{1}\left(\frac{1}{2} ; \frac{n}{2} ; i Z\right),
$$

where $Z$ is a symmetric matrix and ${ }_{p} F_{q}$ stands for a generalized hypergeometric function of matrix argument (defined in James [5]). It follows that the characteristic function for the stationary distribution of the unoriented shape-and-size diffusion, is given by

$$
\begin{aligned}
\Psi_{\widetilde{S}}(Z) & =\mathbb{E}\left\{\exp \left[i \operatorname{tr}\left(Z V^{\top}(I-G) V\right)\right]\right\}=\exp \left\{i \operatorname{tr}\left(V Z V^{\top}\right)\right\} \Psi_{G}\left(-V Z V^{\top}\right) \\
& =\exp \left\{i \operatorname{tr}\left(V Z V^{\top}\right)\right\}\left[{ }_{1} F_{1}\left(\frac{1}{2} ; \frac{n}{2} ;-i V Z V^{\top}\right)\right] .
\end{aligned}
$$

Evidently, the task of inverting such a characteristic function to obtain an explicit form for the distribution is not at all simple. The stationary distribution of the unoriented shape process is then obtained as the restriction of the stationary distribution of the unoriented shape-and-size process to the set of symmetric non-negative definite $k \times k$ matrices $P$ such that $P \mathbf{1}=0$ and $\operatorname{tr}(P)=1$.

Returning to the stationary distribution of the unoriented shape-and-size process, one can see that although there seems little hope of obtaining an explicit form for its distribution, it is possible (in fact, straightforward) to obtain its mean and covariance. In particular, one can take advantage of the fact that if $N \sim \mathcal{N}_{n}\left(0, I_{n}\right)$, then $N /\|N\| \sim \mathcal{U}\left(\mathbb{S}^{n-1}\right)$ to obtain (e.g. Watson [23]),

$$
\begin{gathered}
\mathbb{E}[\tilde{S}]=V^{\top} V-V^{\top} \mathbb{E}[G] V=\frac{n-1}{n} V^{\top} V, \\
\mathcal{C}(\operatorname{vec}(\tilde{S}))=\left(V^{\top} \otimes V^{\top}\right) \mathcal{C}(\operatorname{vec}(G))(V \otimes V),
\end{gathered}
$$

where $\mathcal{C}(\operatorname{vec}(G))$ is the covariance of $G=\left\{g_{i j}\right\}$ with

$$
\begin{aligned}
\operatorname{var}\left(g_{i i}\right) & =\frac{2(n-1)}{n^{2}(n+2),} \\
\operatorname{var}\left(g_{i j}\right) & =\frac{1}{n(n+2)}, \\
\operatorname{cov}\left(g_{i i}, g_{j j}\right) & =-\frac{2}{n^{2}(n+2)},
\end{aligned}
$$

and all other covariances being zero.

Hence, there is a very simple explicit connection between the unoriented shape-and-size of the original ensemble of points (i.e. the shape-and-size modulo reflections) and the mean of the corresponding stationary unoriented Radon shape-and-size diffusion. As in the planar case, this may be exploited to provide a statistical inversion of the unoriented shape-and-size Radon diffusion, for example, through the use of an appropriate ergodic theorem.

Remark 1. A standard procedure in shape theory is to apply a Helmert transformation $Q$ of appropriate dimension to $V$ (from the right) and obtain $W=V Q$. The effect of this transformation is to reduce the dimensionality of the problem. The idea is that knowledge of the centroid of $k$ labelled points, along with $k-1$ of the points suffices to determine 
the ensemble. But in our case the centroid is known to be zero, so that $k-1$ points only will suffice. The first column of $W$ will thus be zero, and so $W$ can be identified with its remaining non-zero columns, so that we move from an $n \times k$ matrix $V$ to a $n \times(k-1)$ matrix $W$. All the results and formulae in this paper hold, of course, if $V$ is replaced by $W$.

\section{Concluding remarks}

When one deals with arbitrary ensembles in arbitrary dimensions, the complexity of shape spaces increases dramatically, and, consequently, so does the geometry of Radon diffusions. It has been shown that one can use the special nature of Radon shape diffusions in order to introduce a straightforward coordinate system, through the use of inner product coordinates and hyperspherical Brownian motion.

Apart from being conceptually straightforward, the representation is advantageous in terms of the simulation of unoriented Radon shape diffusions. In fact, in the physically most significant case (that of ensembles in $\mathbb{R}^{3}$ ) one has that spherical Brownian motion solves the equation

$$
d \beta=\beta \times d B-\beta d t,
$$

where $\{B\}$ is $\operatorname{BM}\left(\mathbb{R}^{3}\right)$ and ' $x$ ' is the cross product (Price and Williams [18]), an equation which is linear in $\beta$, further simplifying simulation.

The inner product representation allowed us to answer some basic questions. The line defined by the hyperspherical Brownian motion was shown to be in bijective correspondence with the unoriented shape (and unoriented shape-and-size) process. It was further shown that the general unoriented Radon shape and shape-and-size processes were diffusions, and Itô equations were derived for the unoriented shape-and-size diffusion. Finally, the parametrization allowed the use of matrix distribution theory to derive the characteristic function for the stationary distribution of the unoriented shape-and-size diffusion. It was seen that this distribution was very simply connected to the unoriented shape-and-size of the generating ensemble.

Of course, in a practical situation such as the one described in Section $2 \cdot 2$, the size is actually observable and of interest, so that talking about strict shape is perhaps not practically significant. However, the restriction of the process to size unity has some interest in terms of the geometry of shape space. In particular, Proposition 1 suggests that the range of the unoriented shape diffusion is diffeomorphic to $n$-dimensional real projective space $\mathbb{R} \mathbb{P}^{n}$. In the planar case, it was seen that the range of the shape-and-size diffusion is an ellipse, while the range of the shape diffusion is a circle. One might thus ask what more can be said about the geometry of the range of the unoriented Radon shape diffusion.

Acknowledgments. The late Professor John Lewis taught me probability theory at Trinity College Dublin. He was a most inspiring teacher. This paper is dedicated to his memory.

I would like to thank Professor David Brillinger for constructive conversations and comments. I would also like to thank a referee for his careful comments.

This research was supported by an NSF Graduate Research Fellowship and an NSF VIGRE fellowship.

[1] T. K. CARne. The geometry of shape spaces. Proc. London Math. Soc. 61 (1990), 407-432. 
[2] R. M. GLAESER. Review: electron crystallography: present excitement, a nod to the past, anticipating the future. J. Struct. Biol. 128 (1999), 3-14.

[3] R. M. Glaeser, W. Chiu, J. Frank, D. DeRosier, W. Baumeister and K. Downing. Electron Crystallography of Biological Macromolecules (Oxford University Press, 2007).

[4] I. L. DRYDEN and K. V. MARDIA. Statistical Shape Analysis (Wiley, 1998).

[5] A. T. JAMES. Distributions of matrix variates and latent roots derived from normal samples. Ann. Math. Stat. 35 (1964), (2), 475-501.

[6] D. G. Kendall. The diffusion of Euclidean shape. Adv. App. Prob. 9 (1977), 428-430.

[7] D. G. Kendall, D. BARDEN, T. K. CARNE and H. LE. Shape and Shape Theory (Wiley, 1999).

[8] W. S. Kendall. Stochastic differential geometry: an introduction. Acta Appl. Math. 9 (1987), 29-60.

[9] W. S. Kendall. The Euclidean diffusion of shape. In Disorder in Physical Systems, ed. G. Grimmett and D. Welsh (Cambridge University Press, 1990), 203-217.

[10] W. S. Kendall. A diffusion model for Bookstein triangle shape. Adv. App. Prob. 30 (1998), 317334.

[11] H. LE. On geodesics in Euclidean shape spaces. J. London Math. Soc. (2), 44 (1990), 360-372.

[12] H. LE and D. G. Kendall. The Riemannian structure of Euclidean shape spaces: a novel environment for statistics. Ann. Stat. 21 (1993), (3), 1225-1271.

[13] K. V. MARDiA and C.G. KhATRI. Uniform distribution on a Stiefel manifold. J. Multivariate Anal. 7 (1975), 468-473.

[14] F. Mosteller and J. W. Tukey. Data Analysis and Regression: a Second Course in Statistics (Addison-Wesley, 1977).

[15] B. ØKSENDAL. Stochastic Differential Equations (Springer, 2003).

[16] V. M. Panaretos. The diffusion of Radon shape. Adv. App. Prob. 38(2) (2006), 320-335.

[17] V. M. PANARETOS. Representation of Radon shape diffusions via hyperspherical Brownian motion. Tech. Report \#707, Department of Statistics, UC Berkeley (April 2006).

[18] G. C. PRICE and D. Williams. Rolling without slipping, I. In Séminaire de Probabilités XVII1981/82 (Paris), Lecture Notes in Mathematics 986 (Springer-Verlag, 1983)

[19] L. C. G. Rogers and D. Williams. Diffusions, Markov Processes and Martingales. Volume 2: Itô calculus. Cambridge Mathematical Library (Cambridge University Press, 2000).

[20] C. G. SMALl. The Statistical Theory of Shape (Springer, 1996).

[21] D. Stoyan, W. S. Kendall and J. Mecke. Stochastic Geometry and its Applications (Wiley, 1995).

[22] M. VAn Den Berg and J. T. Lewis. Brownian motion on a hypersurface. Bull. London Math. Soc. 17 (1985), 144-150.

[23] G. S. Watson. Statistics on Spheres (Wiley, 1983). 